\title{
¿Qué requerimos para una industrialización sostenible del Perú? Una propuesta del modelo industrial
}

\author{
José Porlles Loarte* \\ Orestes Cachay Boza** \\ Gilberto Salas Colotta***
}

\section{RESUMEN}

Diversos especialistas pronosticaban el término del ciclo de bonanza basada en la explotación de las materias primas, en particular de los minerales. El Perú ha sido afectado por esta situación poniendo en evidencia una vez más que el actual modelo primario exportador no proporciona un desarrollo sostenible en el largo plazo. En este contexto de desaceleración de nuestra economía, consideramos que el proceso de industrialización de nuestros recursos es la alternativa más ventajosa que debería emprender el país. Al respecto, el presente trabajo es un aporte de ideas para impulsar un cambio del modelo industrial que debe contar el Perú, para lo cual, en el análisis se ha tomado en cuenta diversa documentación de entidades reconocidas y vinculadas a estos temas.

Palabras clave: competitividad, complejidad, desaceleración, industrialización

WHAT WE NEED FOR A SUSTAINABLE industrialization of Peru? A proposal of the INDUSTRIAL MODEL

\section{ABSTRACT}

Several experts were predicting the end of the cycle of prosperity based on the exploitation of raw materials, especially minerals. Peru has beenaffected by this situation highlighting once again that the current primary export model does not provide a sustainable development in the long term. In this context of a slowdown in our economy, we believe that the industrialization of our resources is the most advantageous alternative to be undertaken by the country. In this respect, the present work is a contribution of ideas to promote a change of the industrial model should have the Peru, for which, in the analysis has taken into account various documents and related entities recognized these issues.

Keywords: competitiveness, complexity, industrialization, slowdown

\section{INTRODUCCIÓN}

"Es importante que todo el mundo entienda que se acabaron los tiempos de las vacas gordas". De esta manera, Lagarde (8) resume la consecuencia de la desaceleración de la economía de China y la imperiosa necesidad que países como el Perú emprendan un ambicioso programa de reformas estructurales.

Voces destacadas en el ámbito económico alertaban de esta preocupación. En el Fórum de la WEF que se realizó en Lima del 25 al 28 de abril del 2013 ya se advertía respecto a la sostenibilidad del crecimiento económico de la región de América latina y por tanto de países similares como el Perú: los precios de los commodities han caído y la demanda interna (base del crecimiento) se va a contraer. En la WEF se advirtió que esa excesiva confianza se está convirtiendo en una neblina que no deja a los países de la región ver los baches que le esperan unos kilómetros más allá (10).

A pesar del crecimiento del producto mostrado por Perú en el último decenio, no

llegamos a ser un país industrializado ni por asomo (4). En la reunión de la ONUDI realizada en Lima sobre la industrialización se destacó que por la diversidad de recursos naturales y materias primas que posee el Perú, a lo largo de nuestra historia hemos atravesado diferentes "booms" económicos (salitre, guano, caucho, harina de pescado, minerales), pero ninguno ha logrado generar esa condición productiva que ha sido el tema central de la reunión. El consenso es que tomado la senda de la industrialización el crecimiento económico se hace más sostenido, generando mayores rentas para el país, así como mejores empleos (5).

Los especialistas anotaban, que el Perú debe crecer mínimo $4 \%$ al año sólo para poder absorber la mano de obra que se incorpora al mercado laboral cada año. Si crecemos $3 \%$ el desempleo aumenta.

El entorno exterior actual (brusca caída de los precios de los commodities y unas condiciones de financiamiento más restrictivas) plantea mayores dificultades para que un país

* Magíster en Administración. Ingeniero Químico. Profesor del Departamento de Análisis y Diseño de Procesos, UNMSM. E-mail: joseporlles@yahoo.com

** Doctor en Ingeniería Industrial. Profesor del Departamento de Gestión y Producción Industrial. UNMSM. E-mail: orestescachay@yahoo.es

*** Ingeniero Químico. Profesor del Departamento de Operaciones Unitarias, UNMSM. E-mail: gsalas19@yahoo.es 
exportador de materias primas, como el Perú, crezca al mismo ritmo que el pasado.

En este contexto, el propósito de este trabajo es el siguiente:

1. Dar a conocer en forma resumida los resultados económicos del país en el marco de la economía global.

2. Hacer notar los puntos débiles que no permiten sustentar el crecimiento de la economía en el largo plazo

3. Resaltar el grado de complejidad del aparato productivo en el contexto global y su relación con el grado de competitividad de la Nación

4. Qué hacer para lograr una reconversión industrial sustentado en una nueva estrategia de desarrollo industrial

\section{LA REALIDAD ECONÓMICA PERUANA}

\section{La macroeconomía estaba bien (Ver Gráfica 1)}

- La economía peruana ha tenido un desempeño notable en el transcurso de la última década, que algunos denominaban "El Milagro Peruano", mostrando una tasa del $6,0 \%$ promedio anual en el periodo 2003 al 2012.

- En este periodo ha pasado a ser un caso exitoso de estabilidad y crecimiento con indicadores que destacan nítidamente en el contexto regional, como lo destaca el PRODUCE (11).

Gráfica 1. Radiografía de la macroeconomía del Perú

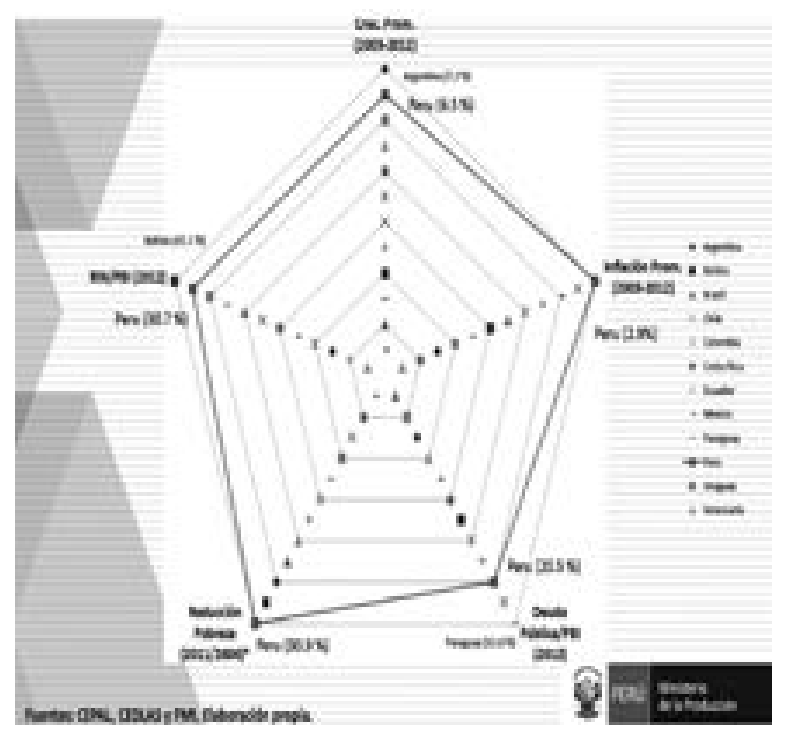

Fuente: PESEM 2011-2015, p. 26.
- Así, entre los años 2003 y 2012 registró la menor tasa de inflación promedio, mejorándose notablemente el nivel de pobreza.

\section{Sin embargo, la realidad peruana presenta otros indicadores poco halagüeños}

La evolución de la economía peruana es muy preocupante cuando se consideran las variables que mejor reflejan el desempeño del bienestar del país, accesos y oportunidades. Comparativamente con sus pares en la región y países referentes, el Perú exhibe hoy:

- Bajo nivel de productividad. Según The Conference Board en el periodo del 2011 al 2014 el indicador PTF ha disminuido 7.8\%.

- Alta presencia de empleo informal, gran desigualdad en los ingresos.

- Bajo nivel de innovación: El promedio de gastos en I+D en América Latina es de $0.7 \% / \mathrm{PBI}$, en tanto que el Perú registra $0.15 \%$.

- Débil institucionalidad (desconfianza en el Poder judicial, Parlamento...).

- El peso de la manufactura no pasa del 15\%/PBI en los últimos 20 años, en tanto este parámetro se exhibe por encima del $20 \%$ en los países emergentes.

- Una estructura productiva que configura a su vez una canasta exportadora con bajo contenido tecnológico y concentrado en recursos naturales.

Entonces la pregunta que se deriva de esta aparente situación es: ¿A qué se debe el crecimiento del Perú en el último decenio?

La respuesta que todos coinciden es al impulso de la inversión privada a la explotación de los recursos naturales, centrada en la minería, aprovechando la subida de precios por la mayor demanda de países como China y, de la agricultura ante mejores perspectivas del Perú al contar con productos que contienen activos funcionales muy buenos para la salud.

Según Hausmman (6): “... América Latina ha tenido buena suerte. La mayoría de los países de la región no ha aprovechado ese ciclo de prosperidad aún para dar el gran salto a una economía generadora de valor agregado y basada en conocimiento". En efecto, esta situación se visualiza en la Gráfica 2.

La dinámica de crecimiento de la economía peruana está marcada por una fuerte inestabilidad

En efecto, al caer el ciclo del "boom de los precios", la economía inicia una caída: PBI 2013: 5\%; 2014: 
2.4\%; previsión 2015: 4,0\%... y nada garantiza que el país crezca por encima del citado $4 \%$.

EI IPE (7) anota: "Hemos perdido muchas oportunidades. Usualmente se afirma que el Perú perdió entre una y tres décadas entre 1960 y 1990 , cuando en realidad se perdió grandes oportunidades, para realizar una transformación productiva" La Gráfica 3 es explicativa en su contenido.

\section{¿Qué explica estas importantes brechas? $Y$} ¿Por qué no nos hemos industrializado?
Variadas razones se esgrimen: hay incentivos no adecuados; no existe capital de riesgo para nuevos emprendimientos, ausencia de una política industrial, entre otros; pero hay otro muy importante: contamos con una estructura productiva desequilibrada.

A pesar del crecimiento de la industria en los últimos años, la diversificación productiva es débil y su peso no es importante en el producto, lo cual también se aprecia en la estructura de nuestras exportaciones (Ver Gráfica 4).

Gráfica 2. Crecimiento del producto, las exportaciones y el peso de la manufactura en el PBI del Perú.

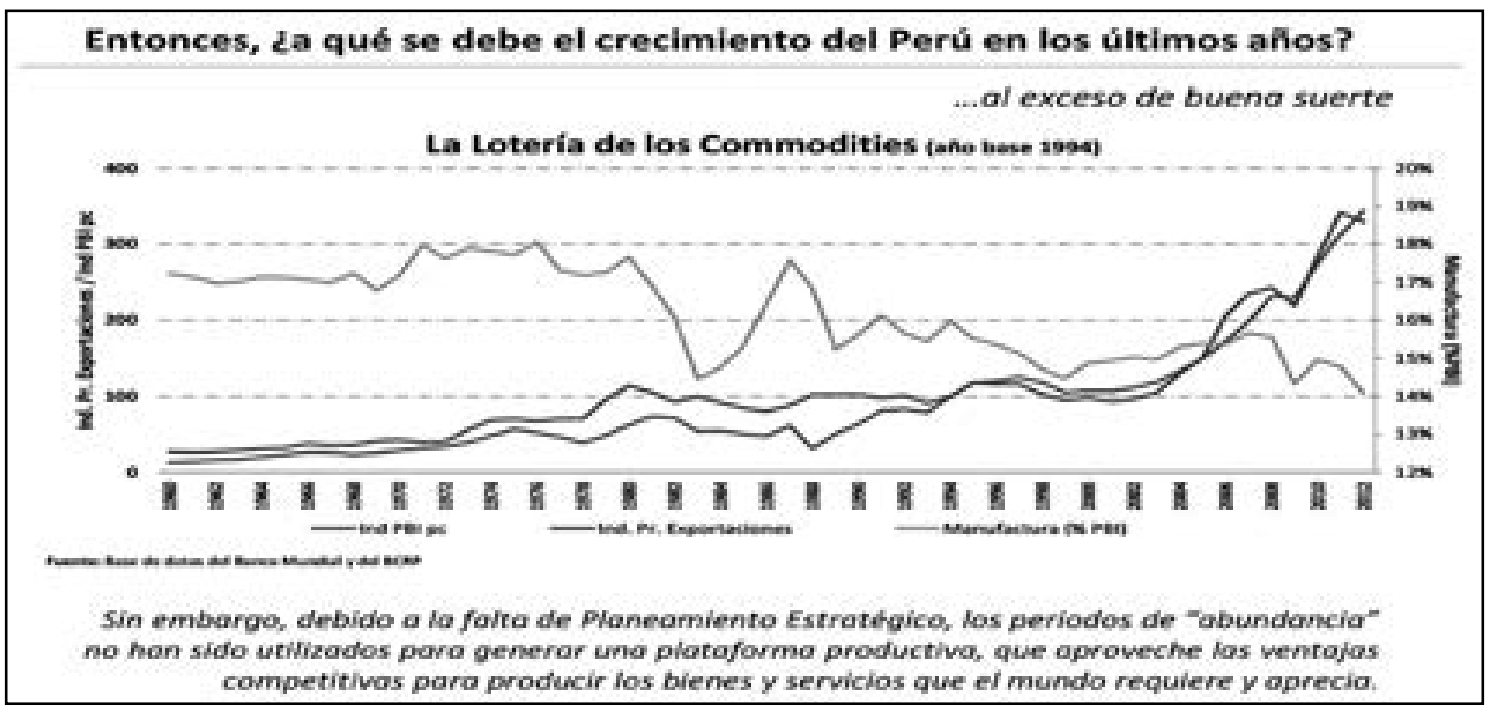

Fuente: CEPLAN. Anderson, C. Presidente CEPLAN. Propuesta de Industrialización. Abril 2014.

Gráfica 3. Proceso de desarrollo del Perú en relación con países referentes.

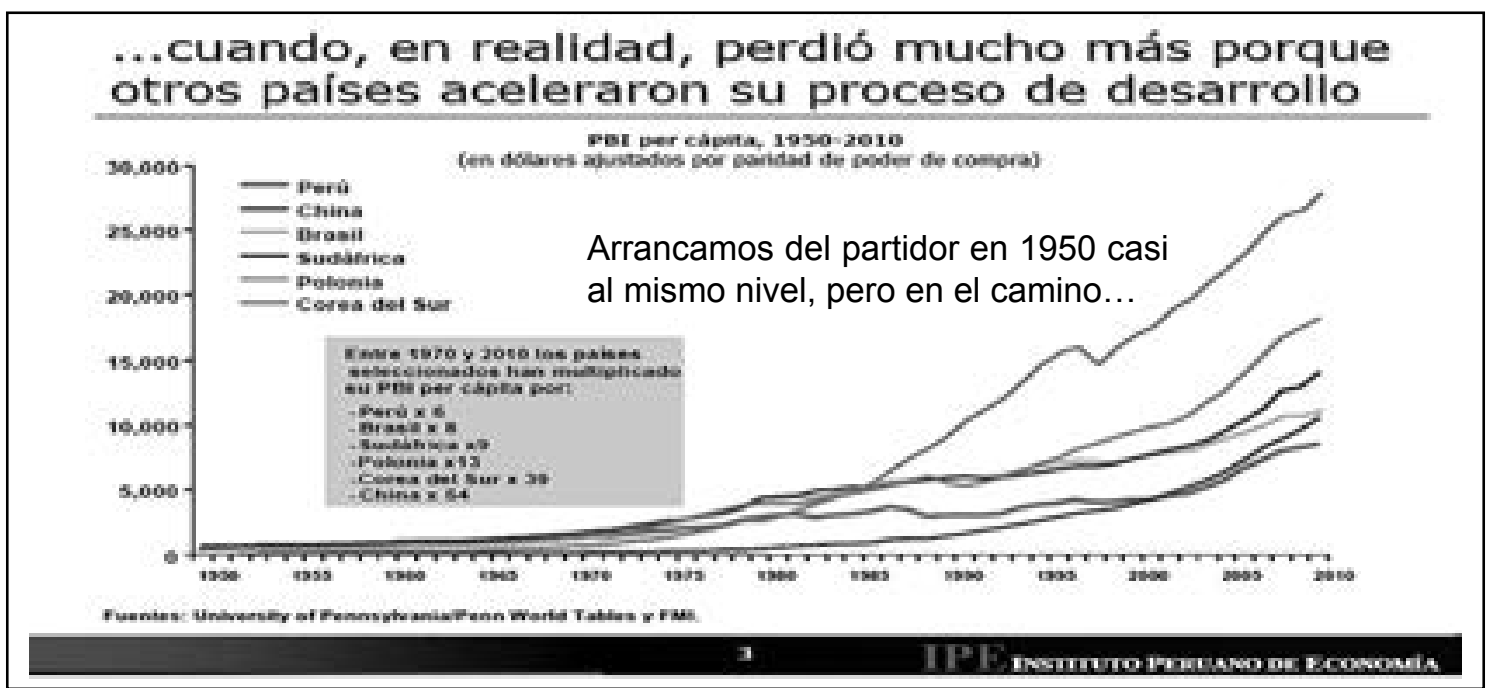


Por ejemplo: Productos primarios: minerales y agro; mmanufactura basada en RN: cátodos de cobre; mmanufactura de baja tecnología: alambrón de cobre.

En el período 2002-2009 la industria creció anualmente al $5.3 \%$, frente al $2 \%$ observado en el período 1995-2001.

Los expertos cuestionan el hecho de que en el tiempo no se hayan desarrollado actividades más intensivas en conocimiento y tecnologías de punta, como es el caso de la producción de software, maquinarias y equipos, química pesada, química de los metales, metalurgia, biotecnología, entre otras actividades basadas en la transformación intensiva de nuestros recursos.

Nuestra diversificación ha estado centrada en algunos sectores de baja tecnología. En la Gráfica 5 se observa la estructura del Sector Industrial desde el punto de vista del VAB: centrada en las tres primeras actividades.

Para entender por qué no despegamos, veamos la ubicación del Perú en complejidad económica (Ver Gráficas 6 y 7). De hecho, las estructuras productivas de las principales economías del mundo tienden a concentrarse en la producción de bienes y servicios de alto valor agregado, intensivos en conocimiento e innovación_tecnológica.

Países latinoamericanos como Brasil, Costa Rica, Colombia, Chile y México le llevan amplia ventaja al Perú en esa senda. Perú se ubica en el cuadrante inferior izquierdo en complejidad económica en el periodo 1972-1910 (Gráfica 6) y en la Gráfica 7 se

Gráfica 4. Estructura de las exportaciones peruanas según contenido tecnológico.

Con exportaciones de muy bajo contenido tecnoliogico.

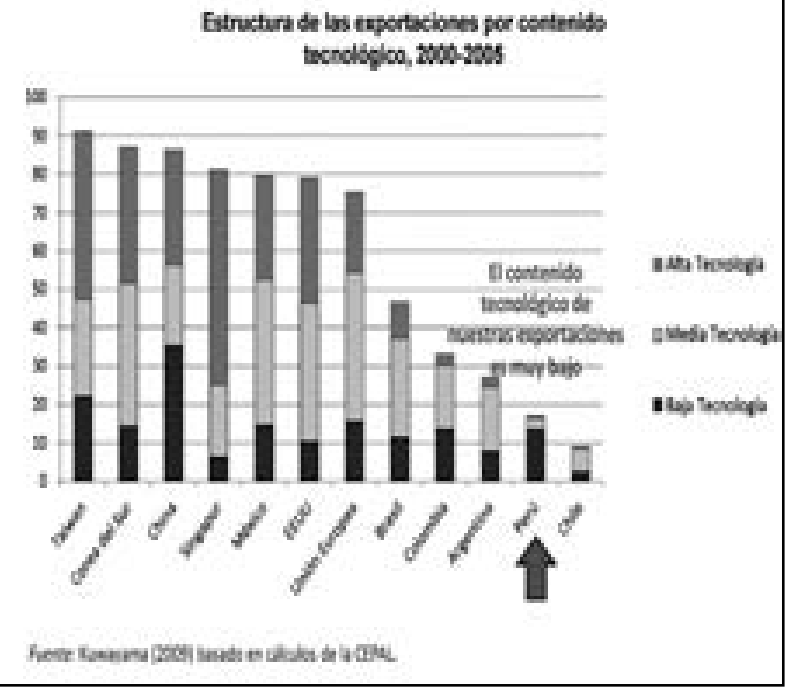

aprecia una relación directa entre esta complejidad económica y el ingreso per cápita del país. Países con altos niveles de ingreso cuentan con productos de muy alto contenido tecnológico.

Lo anterior muestra que el débil proceso de diversificación productiva de nuestro país refleja también una pérdida de terreno en productividad frente a otros países emergentes que sí han tenido una política clara de transformación industrial.

En este marco de análisis, Seminario y otros (13) destacan en su estudio que es peligrosa la dependencia extractiva y que el actual modelo económico es insostenible, sugiriendo mayor diversificación e industrializar nuestros recursos con mayor valor agregado.

\section{Importancia del valor agregado de los productos}

Según el BID, Chile y Costa Rica son las dos economías de la región que usan mejor sus recursos productivos. El rezago del Perú en diversificación y productividad obedece a que, no ha tenido una política clara y estable de desarrollo productivo, cimentada en un consenso con la comunidad económica, social y política del país.

En efecto, en la Gráfica 8 se puede visualizar el peso de la manufactura en el comercio mundial. En tanto en promedio la estructura de exportaciones del Perú es $70 \%$ : productos primarios y $30 \%$ : productos no tradicionales, en el mundo es todo lo contrario.

Gráfica 5. Estructura del sector industrial.

\section{Estructura del Sector Industrtal (Por Gerenoin al Vab)}

\begin{tabular}{|c|c|c|}
\hline \multirow{2}{*}{ Rubres faturiss } & \multicolumn{2}{|c|}{ Part\% } \\
\hline & 1991 & 2009 \\
\hline Intustila Ainerts, Bebles y Tracoco & 31,7 & 32,0 \\
\hline Intustia Quinia, Cacho y Pistion & 12,6 & 19,7 \\
\hline 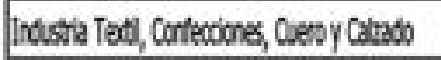 & 19,7 & 10,3 \\
\hline 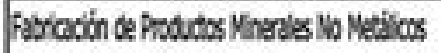 & 5,5 & 10,1 \\
\hline fatrocitin Produds Metiles & 11,0 & 8,4 \\
\hline Intustias Netallas Bisicas & 7,1 & 8,1 \\
\hline Intststia dat Paped, Inpreta y Esción & 5,1 & 7,3 \\
\hline haductos Nenfacturads Dherss (Mudes) & 3,4 & 23 \\
\hline Intustra Maser y Moderales & 40 & 1,8 \\
\hline
\end{tabular}


Gráfica 6. Complejidad económica periodo $1972-$ 2010.

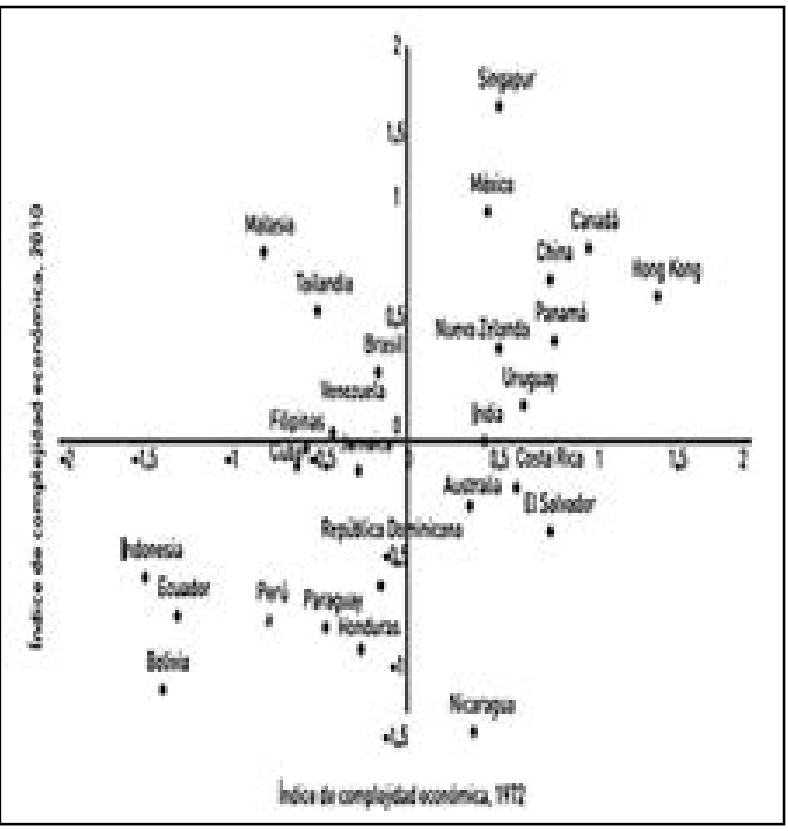

Fuente: PESEM 2011-2015, página 26

Gráfica 7. Relación positiva: complejidad económica y crecimiento económico.

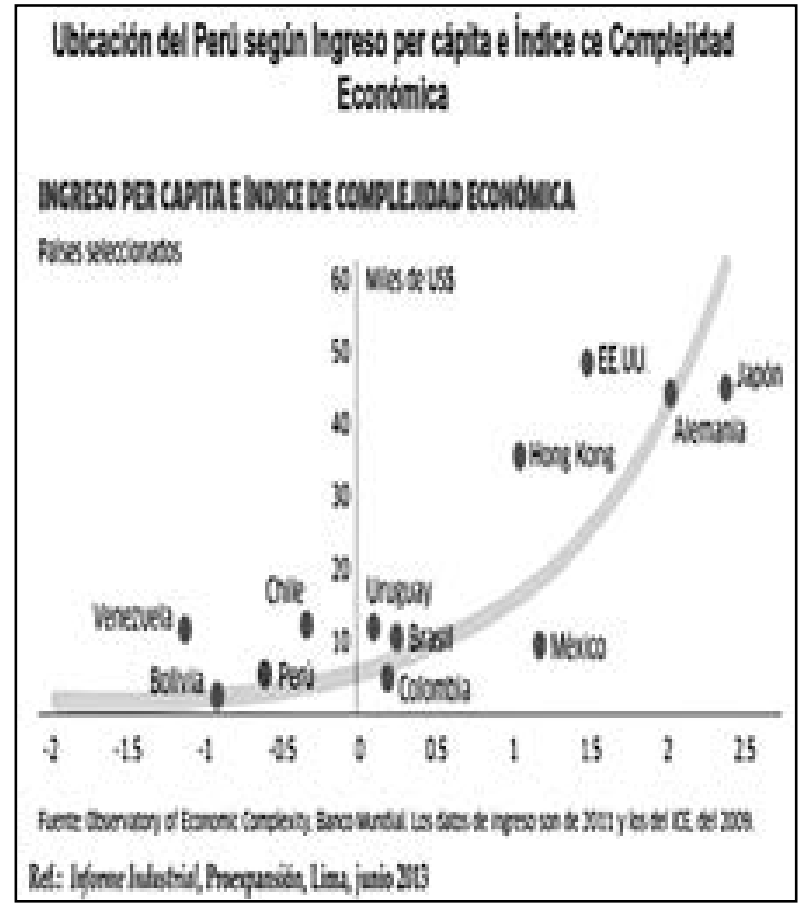

Fuente: El Plan Nacional de Diversificación Productiva Peruano y la nueva orientación en el BID. Luyo, Jaime. UNI. Setiembre de 2014. http://www.eumed.net/cursecon/ecolat/pe/2014/produccion.html
Gráfica 8. Participación de la manufactura en el comercio mundial.

\section{La manufactura domina el comercio mundial}

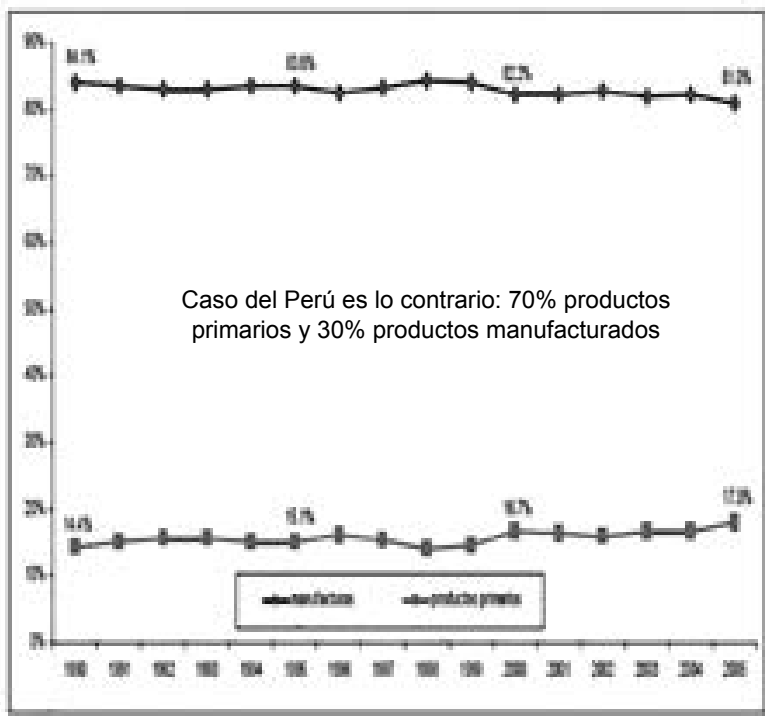

Fuente: Fuente: PESEM 2011-2015, página 21

Más aún en la Gráfica 9 se evidencia que dentro de las manufacturas, el comercio se centra en los productos con alto contenido tecnológico.

Gráfica 9. Contenido tecnológico en el comercio de la manufactura.

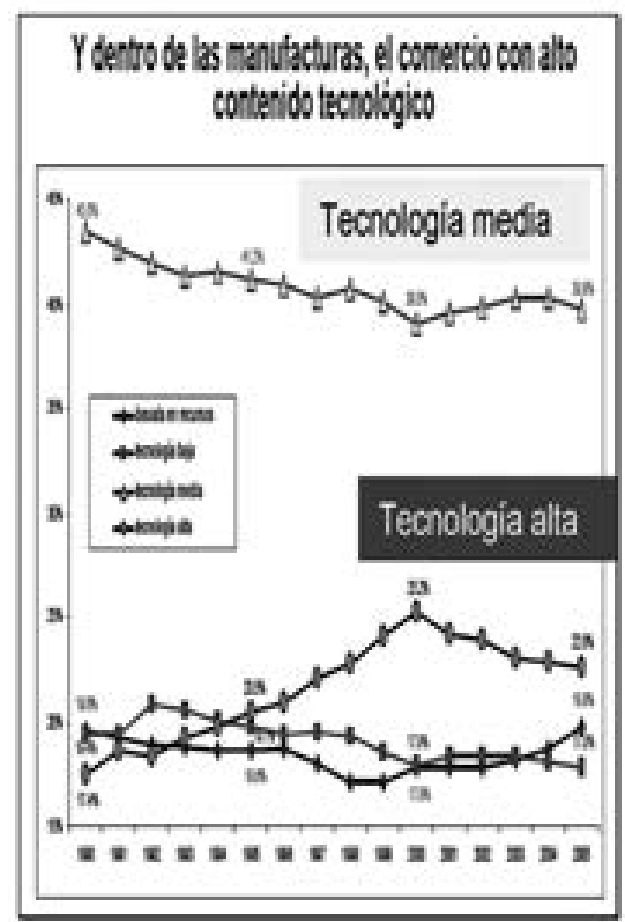

Fuente: Fuente: PESEM 2011-2015, p. 21. 


\section{¿HA SIDO BENEFICIOSO LA ESTRATEGIA DE CRECIMIENTO SEGUIDA POR PERÚ?}

Como se aprecia en el Cuadro 1, mientras la estructura productiva de Corea del Sur ha crecido progresivamente en sofisticación al aumentar la participación de productos más complejos en su canasta exportadora, el grado de sofisticación de la economía peruana es bajo y se ha mantenido relativamente estable en los últimos 25 años.

En la medida que la economía peruana ha sostenido una estrategia de crecimiento basada en la explotación de recursos naturales, la evolución favorable de los precios internacionales de los minerales en la década previa ha permitido al país crecer rápidamente y resolver muchos de los problemas relevantes que lo aquejaban.

Sin embargo, la evidencia empírica muestra que mantener esta estrategia de crecimiento en el largo plazo, sin modificaciones o complementos, puede ser problemático por variadas razones.

\section{Las fases de industrialización versus el curioso caso peruano.}

Según CEPLAN (1) nuestro país representa una economía desequilibrada y difícil de conceptualizarla en cuando al grado de desarrollo industrial, como se visualiza en el Cuadro 2. Según la participación en la manufactura el modelo de industrialización es primario exportador; si se considera el nivel de riqueza, estamos casi a la par con países emergentes; en tanto si se mide la participación del PEA del sector servicios es una economía post industrializada.

No obstante lo anterior, a juicio nuestro el país al no contar con industrias relevantes, configura uno que recién estaría ingresando a un proceso de industrialización.

\section{¿QUĖ ES LA COMPETITIVIDAD: ¿POR QUĖ LA OBSESIÒN DE LOGRARLO?}

Conceptualmente competitividad implica:

- Capacidad (contar con ventajas competitivas)

- Para crecer (aumentar el nivel de riqueza)

- En el largo plazo y de manera sostenible

Según la WEF (14) la competitividad es: "La medida relativa en que un país crea las condiciones económicas, sociales y medioambientales para sentar las bases del desarrollo sostenible". La WEF anualmente evalúa a los países y realiza un ranking denominado: Índice de Competitividad, para lo cual considera 12 parámetros. Este informe es de suma importancia, por cuanto es un referente para los inversionistas del mundo.

\section{¿Què tan competitivos somos? (Ver Cuadro 3)}

En los últimos 10 años, Chile es el país más competitivo en América Latina, pero comparte posiciones con Puerto Rico en América Latina y El Caribe. Perú registra drástico retroceso, resultado de la situación de convulsión social y manejo del mercado con un modelo que se torna inadecuado para los intereses nacionales.

Cuadro 1. Exportaciones por contenido Tecnológicos de países referentes (En \% del total de exportaciones).

\begin{tabular}{|c|c|c|c|c|c|c|}
\hline \multirow[b]{2}{*}{ Pais } & \multicolumn{2}{|c|}{$\begin{array}{c}\text { Baja } \\
\text { tecnologia }\end{array}$} & \multicolumn{2}{|c|}{$\begin{array}{l}\text { Media } \\
\text { tecnologia }\end{array}$} & \multicolumn{2}{|c|}{$\begin{array}{c}\text { Alta } \\
\text { tecnologia }\end{array}$} \\
\hline & $\begin{array}{l}7990 . \\
1995\end{array}$ & 2005 & $\begin{array}{l}1990=- \\
1995\end{array}$ & 2005 & $\begin{array}{l}1990:- \\
1995\end{array}$ & 2005 \\
\hline Peñ & 11.7 & 13.6 & 2.5 & 3.0 & 0.5 & 0.6 \\
\hline Mexico & 12.9 & 14.6 & 37.3 & 37.9 & 17.5 & 27.3 \\
\hline $\begin{array}{l}\text { Cosia } \\
\text { Rica }\end{array}$ & 12.8 & 15.7 & 7.7 & 163 & 3.4 & 30.7 \\
\hline Corea & 29.3 & 74.3 & 34.3 & 37.4 & 25.8 & 35.4 \\
\hline Crina & 49.5 & 352 & Tा.T & 212 & 110 & 30.0 \\
\hline Singapur & 8.1 & 6.4 & 220 & 18.8 & 45.4 & 559 \\
\hline
\end{tabular}

Fuente: Kunayama, 2009.

Cuadro 2. Categorización de la economía peruana.

\begin{tabular}{|c|c|c|c|}
\hline \multicolumn{4}{|c|}{ 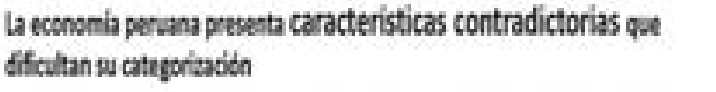 } \\
\hline belador & Nwel & Coxifcricton & $\begin{array}{l}\text { twononix } \\
\text { Simlares }\end{array}$ \\
\hline 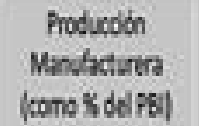 & $15 \%$ & $\begin{array}{l}\text { Prosouclibe } \\
\text { Pinaria }\end{array}$ & $\begin{array}{l}\text { Alanishin } \\
\text { sctowas } \\
\text { Seropil }\end{array}$ \\
\hline Plos cipta & USS 6,018 & Inereso Medo & $\begin{array}{l}\text { Oind } \\
\text { Matat } \\
\text { Prand }\end{array}$ \\
\hline 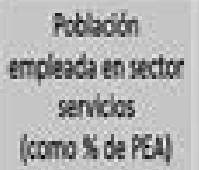 & 74.85 & $\begin{array}{l}\text { Econonia fost- } \\
\text { industrialuda }\end{array}$ & $\begin{array}{l}\text { Sucha } \\
\text { Sin } \\
\text { Kepoth } \\
\text { Rero Unide }\end{array}$ \\
\hline
\end{tabular}

Fuente: Fuente: CEPLAN. Anderson, C. Presidente CEPLAN. Propuesta de Industrialización. Abril 2014. 
Cuadro 3. Informe Îndice de Competitividad Global

\begin{tabular}{cccc}
\hline País & $\mathbf{2 0 1 2 -}$ & $\mathbf{2 0 1 3 -}$ & $\mathbf{2 0 1 4 -}$ \\
& $\mathbf{2 0 1 3}$ & $\mathbf{2 0 1 4}$ & $\mathbf{2 0 1 5}$ \\
\hline Suiza & 1 & 1 & 1 \\
Singapur & 2 & 2 & 2 \\
EEUU & 7 & 5 & 3 \\
Finlandia & 3 & 3 & 4 \\
Corea & 19 & 25 & 26 \\
China & 29 & 29 & 28 \\
Chile & 33 & 34 & 33 \\
Colombia & 69 & 69 & 66 \\
México & 53 & 55 & 61 \\
Argentina & 94 & 104 & 104 \\
Perú & 61 & 61 & 65 \\
Brasil & 48 & 56 & 57 \\
Puerto Rico & 31 & 30 & 32 \\
\hline
\end{tabular}

Fuente: WEF. Elaboración propia.

Registramos bajos niveles de ventajas competitivas en (Ver Cuadro 4): Innovación, Investigación, educación, Adopción tecnológica e Instituciones

\section{¡ESTAS SON NUESTRAS MAYORES DEBILIDADES!}

Al respecto, en relación a nuestro país en el informe del CDI-SIN (3), el World Economic Forum menciona: "A pesar de la caída de cuatro posiciones, Perú sigue en la mitad superior de la clasificación. Las preocupaciones sobre el funcionamiento de las instituciones (118), junto con el insuficiente progreso en la mejora de la calidad de la educación (134) y adopción tecnológica (92), explican el descenso del país.

Menciona que Perú recientemente se ha beneficiado de un fuerte crecimiento gracias al aumento del precio de los minerales.

El país necesita fortalecer sus instituciones públicas (127) mediante el aumento de la eficiencia del gobierno (116), luchar contra la corrupción (103), y mejorar la infraestructura (88). Además, desarrollar la capacidad para generar y utilizar el conocimiento y así diversificar la economía hacia actividades más productivas y que requerirán elevar la calidad de la educación (134), ya que aún no es capaz de proporcionar las habilidades necesarias para una economía cambiante; también impulsar la adopción de tecnología (92), que incluye una mayor aceptación y uso de las TIC (101); y elevar su capacidad de innovación (117), que sigue siendo baja. Estas acciones requieren tiempo para desarrollarse y dar frutos."
Cuadro 4. Evolución del Perú en los 12 pilares de la Competitividad

\begin{tabular}{|c|c|c|c|c|c|}
\hline & \multirow{2}{*}{ PILAES } & \multicolumn{2}{|c|}{20142015} & \multicolumn{2}{|c|}{2013.2014} \\
\hline & & Posicón & Vlor & Posión & Valor \\
\hline 1 & hetecones & 188 & 33 & 100 & 34 \\
\hline 2 & Ifrestudter & 88 & 35 & 91 & 35 \\
\hline 3 & Eltumo Nacroceonónico & 21 & 59 & 20 & 5.9 \\
\hline 4 & Stad y odcacion pingris & H & 54 & 96 & 54 \\
\hline 5 & 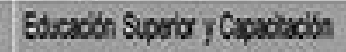 & 8 & 4.1 & 88 & 40 \\
\hline 6 & Elcencis dal necudo da Seres & 53 & 45 & 52 & 4.4 \\
\hline 7 & Elisencia del merado labori & 51 & 43 & 48 & 4.5 \\
\hline 8 & Desando da macsob franciero & 40 & 45 & 4. & 4.5 \\
\hline 9 & Preparaciba teroblogica & $\$$ & 33 & 8 & 34 \\
\hline 10 & Tanato de nercado & 43 & 45 & 43 & 45 \\
\hline$\pi$ & Solisticacion enpresaid & $n$ & 39 & 74 & 39 \\
\hline 12 & movacion & 117 & 28 & 122 & 28 \\
\hline
\end{tabular}

Fuente: WEF. Elaboración: CDI-SIN, 2014.

La competitividad de los países y la necesidad de contar con una estructura industrial hacia la manufactura con mayor valor agregado

Así lo señalan los expertos en estos temas. El análisis realizado por Hausmann (2011) grafica que existe una relación directa entre el nivel de complejidad tecnológica de los países con su nivel de competitividad, tal como se aprecia en la Gráfica 10. Esta situación abona la tesis de la necesidad de rediseñar el modelo actual de desarrollo, mediante una reingeniería hacia la industrialización bajo las actuales circunstancias.

Porlles (9) anotaba que "Las fórmulas neoliberales aplicadas en el Perú como en ALC para combatir la pobreza y la desigualdad social, basado en el goteo de la prosperidad de la mano invisible del mercado, han fracasado". Adicionaba que existen peligros inminentes:

- Que se acabe la "fiesta de las exportaciones de minerales", que han impulsado el incremento de las divisas y del PBI.

- Aumento exponencial de la delincuencia, convirtiéndose en "una ocupación laboral".

- Que se produzca un desborde social."

Sin ánimos de ser futurólogo, en efecto, se acabó la "época de las vacas gordas generado por la minería" y el país atraviesa además por la peor situación en 
Gráfica 10. Relación complejidad tecnológica y la competitividad de los países.

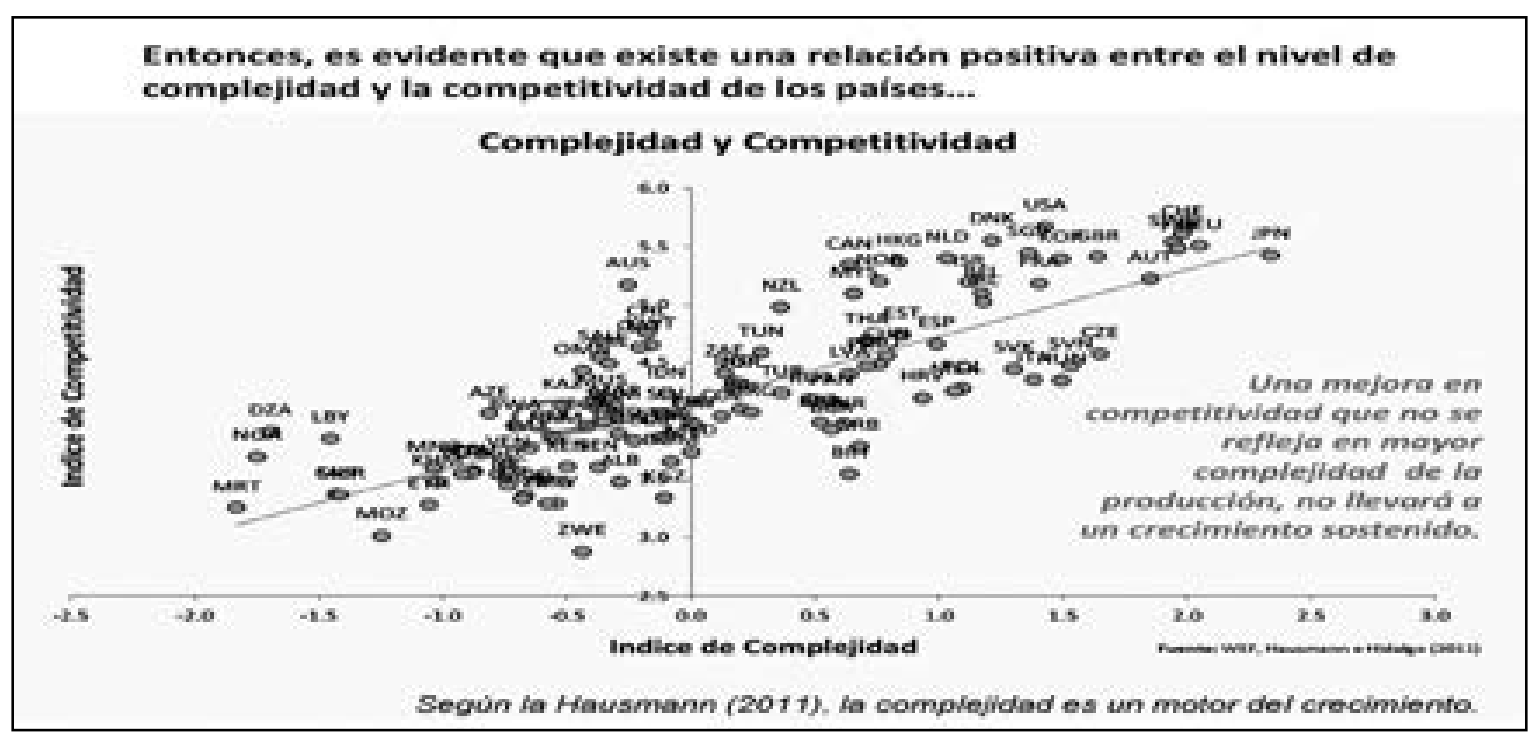

Fuente: Anderson, C. Presidente CEPLAN. El Perú en los Rankings de Competitividad Mundial. Marzo 2014; pp. 11.

el plano delincuencial y seguridad ciudadana, en adición a la caída del producto.

¿La solución?: no es fácil, pero la propuesta global de CEPLAN es posible, PERO NO SUFICIENTE...

CEPLAN (2) propone una producción diversificada, “... mediante un proceso de industrialización ordenado, inclusivo y orientado al futuro que articule a las PYMEs con las Multi-Latinas Peruanas (MLP)", para lo cual diseña lograr conglomerados modernos y PYMEs intensivas en conocimiento.

Es un modelo frágil y no agresivo para las actuales circunstancias que atraviesa el país. Lo único rescatable es la expedición de la Ley 30309 del 12 de marzo del 2015 que mejora el incentivo tributario para el pago del impuesto a la renta de una deducción hasta $175 \%$ para gastos que realicen las empresas en investigación e innovación.

Sin embargo, deja a las fuerzas del mercado el funcionamiento del modelo propuesto. Como ejemplo tenemos que no se propone un diseño territorial de ubicación de las industrias estratégicas, aunque tampoco las define, impulsando más los temas de permisologìa, dando prioridad a la disminución de los costos de transacción. La propuesta no está en línea con lo que recomienda organizaciones como la ONUDI.

Salazar (12) señala: “... Reducir las trabas burocráticas, la permisologìa para abrir una industria e impulsar la infraestructura será elemental para determinar las inversiones. Hablamos de modernización de puertos, desarrollo de la red vial, la longitudinal de la sierra, de infraestructura ferroviaria". Sobre incentivos tributarios, anota que los plantearon, pero que la respuesta del Gobierno fue negativa. Wedemeyer (15) también empresario anota "... si en otros países se brinda a la inversión parques industriales ( $y$ no hay que buscar donde poner una fábrica), el proceso es ágil y hay servicios como energía y comunicación, la inversión irá a esos países".

\section{¿QUÉ HACER? PROPUESTA DEL MODELO DE INDUSTRIALIZACIÒN CON MEDIDAS ESTRATĖ- GICAS Y ESTRUCTURALES DE DESARROLLO}

En este trabajo se propone un primer esquema de desarrollo industrial como línea base, para complementarlo e ir mejorándolo en el futuro.

\section{OBJETIVO}

Establecer una política industrial diferenciada de promoción de inversiones, con metas a lograr resultados concretos.

\section{Líneas estratégicas:}

\section{Construir un Estado}

- EMPRESARIO- PLANIFICADOR: No se puede dejar el orden y las prioridades a la "mano invisible del mercado". El estado prioriza la asignación de recursos en las actividades productivas estratégicas en función de nuestras ventajas comparativas y competitivas.

- PROMOTORDELDESARROLLOINDUSTRIAL: El estado puede promover empresas con cierta 
participación entre el $20 \%$ al $30 \%$ para dar confianza a los inversionistas privados en las actividades priorizadas.

- REGULADOR DEL MERCADO: El estado debe tener una presencia más estricta en estos aspectos, a través de los organismos ya existentes.

2. Establecer incentivos tributarios para la nueva inversión en las industrias estratégicas que el Estado defina

Promover las inversiones orientadas a las exportaciones con INCENTIVOS TRIBUTARIOS, que permitan el desarrollo tecnológico y transformación de nuestros recursos naturales para darle mayor valor agregado en las actividades priorizadas.

Todas las empresas en las actividades priorizadas que reinviertan sus utilidades o las nuevas que se establezcan en el país tendrán la opción de contar con beneficios ttributarios por hasta 10 años contados a partir de la fecha de inicio de operaciones comerciales, con deducción hasta el $100 \%$ de los gastos efectuados en la inversión con cargos a las utilidades reinvertidas o a las futuras utilidades.

3. Conversión de un modelo primario exportador a uno de producción de productos con alto valor agregado o alto contenido tecnológico (el éxito de la competencia futura se dará en estos planos)

Ver en el Cuadro 5 la propuesta de los programas industriales de desarrollo estratégico para el país.

Obtener alto nivel de competitividad sobre la base de una mayor complejidad del aparato productivo industrial.

¿Por qué un nuevo modelo de desarrollo y nuevas formas de pensar con visión externa?
- Tener recursos No es suficiente (No caer en la paradoja "La maldición de los recursos")

- Requerimos construir y proyectar varias cosas:

1. Confianza en nosotros mismos, que sì lo podemos hacer: Cambio de actitud y hacer bien las cosas: con eficiencia.

2. Confianza en los inversionistas nacionales y extranjeros: "Empresarios SI" , "Empresaurios NO"

3. Que no es suficiente producir bien, saber dónde están los mercados y cómo se vende (comercialización)

4. Irradiar al mundo que abrimos las puertas a la inversión mundial como lo ha hecho Singapur, Corea.

5. Confianza y conectividad entre la Universidad y la Empresa para impulsar la INVESTIGACIÓN APLICADA

4. Construcción de nuestras ventajas competitivas en los parámetros que mostramos debilidad.

\section{¿Cómo Hacerlo?}

1. Promover la construcción de parques industriales: conglomerados o clusters, incluyendo toda la cadena productiva (caso de Gamarra...).

El Estado debe ser el promotor activo, definiendo las áreas en donde estarán estas industrias estratégicas, priorizando la construcción de la infraestructura necesaria para el transporte y logística. No dejarlo a las manos invisibles del mercado. También se puede otorgar incentivos tributarios similares a lo planteado en este documento a todas las

Cuadro 5. Programas industriales estratégicos para impulsar el desarrollo industrial del Perú

\begin{tabular}{|c|c|}
\hline \multicolumn{2}{|c|}{ 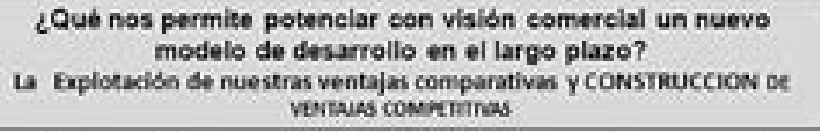 } \\
\hline \multicolumn{2}{|c|}{$\begin{array}{c}\text { Seis grandes gies de desarello, elaves Pare una transformación } \\
\text { industrial organiwada con'ALTO VALCRACRECADO }\end{array}$} \\
\hline Recursos & $\begin{array}{c}\text { Prognama Industriallearques } \\
\text { Industrislos? }\end{array}$ \\
\hline Gas de Camisea & 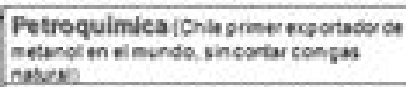 \\
\hline legumbres, maderas. & 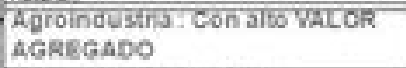 \\
\hline Yacimiantos y minerales & Quimica de los metales \\
\hline $\begin{array}{l}\text { Lana Alpaca y Algodón da } \\
\text { callesd }\end{array}$ & Textiles y confocelones \\
\hline Metales & Metalurgia y MetalMecinica \\
\hline Turismo & $\begin{array}{l}\text { Turismo (Aprovechando los sitios } \\
\text { y rutas areueolopicas) }\end{array}$ \\
\hline
\end{tabular}


inversiones en infraestructura para lograr habilitar las áreas de las nuevas industrias priorizadas.

En cuanto a los parques industriales, en adición a los ya existentes se sugiere lo siguiente:

1. Parque industrial en la Oroya (Química de los metales: la gran industria derivados de sales y pigmentos de cobre, plomo y zinc: corresponde a industrias con alto contenido tecnológico).

2. Parque industrial Metal Mecánico en Arequipa (producción de productos metalúrgicos como bolas de acero, barras y todos los derivados para la minería).

3. Parque industrial en Mollendo (Química del cobre y sus derivados e industria no metálica)

4. Parque industrial Agroindustrial en Trujillo, Loreto, Ucayali e Ica (producción de derivados de los productos del agro con alto valor agregado).

5. Parque industrial en Lima y Trujillo (producción derivada de la química en general, no metálica, textiles en hilados y acabados de algodón).

6. Parque industrial textil en Arequipa (producción y derivados de textiles de alpaca).

7. Parque industrial para los polos petroquímicos.

\section{Promover la construcción de los parques tecnológicos}

Que son ciudades inteligentes que impulsan la innovación, la investigación científica y tecnológica. Estos parques se desarrollan con la participación del Estado, universidades, centros de investigación y privados, para lo cual también el Estado otorga incentivos tributarios pertinentes.

En estos parques Perú es uno de los pocos países en América del Sur que no cuenta con estos instrumentos de desarrollo de la investigación.

\section{CONCLUSIONES}

1. El Perú no cuenta con un marco favorable para generar una diversificación productiva: hay que construirla. El Estado debe dar forma al mercado.

2. No se aprecia vínculos entre el crecimiento económico y el desarrollo social. El crecimiento "NO CHORREA" en relación a los ingentes beneficios de la inversión minera.

3. Con el actual modelo, SIN LA REQUERIDA INDUSTRIALIZACIÓN, NO se puede contar en el largo plazo con un desarrollo sostenible con EQUIDAD y BIENESTAR
4. URGE UN CAMBO RADICAL DEL MODELO DE DESARROLLO: con un Estado planificador, regulador, promotor y EXIGIENDO la producción $Y$ TRANSFORMACIÓN de lOS RECURSOS NATURALES CON MAYOR VALOR TECNOLÓGICO

5. EL ESTADO DEBE priorizar la construcción de las ventajas competitivas $(I+D)$ a través de las universidades y centros tecnológicos base para lograr un nivel de MODERNIZACION Y DE CAMBIO TÉCNICO.

\section{REFERENCIAS BIBLIOGRÁFICAS}

[1] CEPLAN (2014). Anderson, C. Propuesta de Industrialización, Abril 2104, pp.16-17.

[2] CEPLAN (2014), op. cit., pp. 31-32.

[3] CDI-SIN (2014). Informe de Competitividad Global 2014-2015. www.cdi.pe/IGC.2014_2015.htm

[4] Día 1/El Comercio (2013). Fecha: 9 de diciembre del 2013, pp. 12.

[5] Día 1/El Comercio (2013), op. cit., pp. 12.

[6] Hausmann, R. (2015). Portafolio/El Comercio de fecha 28 de Abril del 2015, pp. 6.

[7] IPE (2013).

[8] Lagarde, Ch. (2015). Directora General del Fondo Monetario Internacional-FMI. Portafolio/ El Comercio del 12 de abril del 2015, pp. 8 y 9.

[9] Porlles, J. (2005). Posición Competitiva y Modelo Productivo en el Perú. Revista de la Facultad de Ingeniería Industrial, Industrial data, Vol. (8): pp. 42-51 (2005) UNMSM.

[10] Portafolio/El Comercio (2013). Fecha 28 de Abril del 2013, pp. 6.

[11] PRODUCE (2014). Plan Nacional de Diversificación productiva. Mayo 2014, pp. 27.

[12] Salazar, L. (2013). Presidente de la Sociedad Nacional de Industrias-SIN. Día 1/El Comercio de fecha 9.12.13, pp.13.

[13] Seminario, B., Alva, N., Sanborn, C y Schuldt, J. $(2$ 013). Cuando Despertemos en el 2062, Visiones del Perú en 50 Años. Universidad del Pacífico. Fondo Editorial CIUP, 2013.

[14] WEF (2015). Wilkipedia. http://es.wikipedia. org/wiki/Foro_Econ\%C3\%B3mico_Mundial.

[15] Wedenmeyer A. (2013); op. cit., pp. 13. 